\title{
Revista

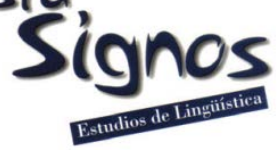

\section{La representación de la revolución en el texto escolar: 'Ser' y Ilegar a ser' en la historia}

\section{The representation of revolution in the school textbook: Being and becoming in bistory}

\author{
Diana Ávila \\ UNIVERSIDAD DEL NORTE \\ INSTITUCIÓN EDUCATIVA TÉCNICA \\ Comercial Francisco Javier Cisneros \\ COLOMBIA \\ dihanyag@gmail.com
}

\author{
Norma Barletta \\ UNIVERSIDAD DEL NORTE \\ COLOMBIA \\ nbarlett@uninorte.edu.co
}

\author{
Diana Chamorro \\ UNIVERSIDAD DEL NORTE \\ COLOMBIA
}

dchamorro@uninorte.edu.co

Recibido: 05-VIII-2015 / Aceptado: 30-VIII-2016

\section{Resumen}

Este artículo analiza cómo la gramática del texto escolar y, más específicamente, la escogencia de verbos de tipo 'ser' y 'tener', denominados procesos relacionales desde una perspectiva sistémico-funcional, puede dificultar la comprensión de los eventos históricos y presentar una visión sesgada de los mismos. Se presenta el análisis de los procesos relacionales y su utilización en el capítulo sobre la Revolución Industrial de un texto de Ciencias Sociales de octavo grado y se discuten las implicaciones para la enseñanza y el aprendizaje. El análisis indica que la escogencia recurrente de los procesos relacionales favorecería una visión de la historia en la cual la mayoría de los fenómenos 'son', 'están' o 'se vuelven' sin la intervención de actores humanos. Una práctica docente despreocupada de esta característica de los libros de texto contribuiría a mantener las desigualdades de los estudiantes en cuanto al manejo del discurso no cotidiano que da acceso al conocimiento científico y los contextos de su uso.

Palabras Clave: Lingüística sistémica-funcional, procesos relacionales, visión de la historia, enseñanza y aprendizaje, textos escolares. 


\begin{abstract}
This paper analyzes how the grammar of school textbooks, more specifically the choice of the verbs 'be' and 'have' - known as relational processes in the systemic-functional framework - may make it more difficult for learners to understand historical events and presents a biased view of these events. We present an analysis of relational processes and their use based on a chapter on the Industrial Revolution from an eighth-grade Social Science textbook and discuss their implications for the teaching and learning of history. The analysis shows that the recurrent use of relational processes promotes a view of history in which most things simply 'are' or 'become' without human intervention. Pedagogical practices which disregard this feature of textbooks play a part in maintaining inequalities between students with respect to dealing with the noneveryday discourses which provide access to scientific knowledge and the contexts in which it is used.
\end{abstract}

Key Words: Systemic-functional linguistics, relational processes, views of history, teaching and learning, textbooks.

\title{
INTRODUCCIÓN
}

El texto escolar es considerado, en muchos contextos, un apoyo fundamental para la enseñanza y el aprendizaje de los contenidos de los diversos saberes, entre ellos los de las Ciencias Sociales (Atienza, 2007; Negrín, 2009; Aguirre, 2015). El libro de texto muchas veces determina el orden, la cantidad y el nivel de profundidad con el cual se va a desarrollar un tema. Se asume que el texto, por una parte, constituye el puente ideal en la transición entre el manejo del lenguaje puramente cotidiano y el lenguaje académico propio de la historia, y por otra, que presenta una visión equilibrada y autorizada de los hechos de las Ciencias Sociales (Oteíza, 2006; Moss, 2010).

El texto escolar se puede considerar como un macro-género (Martin, 1997; Hyland, 2002) en el sentido de que combina géneros más elementales, por ejemplo, en historia tenemos el recuento histórico, informe histórico y explicación histórica (Oteíza, 2006).

Sin embargo, como afirma Short (1994), la enseñanza de la historia implica por lo general una buena cantidad de lectura de textos que son largos y llenos de conceptos abstractos y nuevos para los estudiantes. Eggins, Wignell y Martin (1993) han estudiado los libros de historia de la escuela media en Australia y han demostrado que sus autores utilizan un lenguaje con escogencias lingüísticas que difieren de las usadas en el lenguaje cotidiano. En los libros que ellos estudiaron encontraron que cuando los autores interpretan los hechos y generalizan a partir de ellos, las personas desaparecen y las acciones se cosifican y su secuencia temporal pierde dinamismo. Otro aspecto importante del discurso de la historia es la forma segmentada como se expresa el tiempo (Coffin, 2006), lo que dificulta la comprensión de las alumnas. Afirma Coffin (2006) que sin la capacidad de formar divisiones cronológicas y 
secuenciar eventos, el pasado es un caos para los estudiantes y no puede existir ninguna comprensión real de las nociones de cambio, desarrollo, continuidad, progresión y regresión.

Otras investigaciones muestran que el lenguaje con el que se presentan los hechos históricos representa un reto para los estudiantes, puesto que utiliza registros académicos con abundantes nominalizaciones y uso ambiguo de conjunciones (Martin, 1991; Unsworth, 1999). Beck, McKeown y Gromoll (1989), por su parte, han identificado que los textos no explicitan las conexiones entre las ideas y asumen un conocimiento previo de conceptos abstractos que los estudiantes no tienen, lo cual no permite a los lectores la identificación de los participantes y los eventos.

En el contexto iberoamericano, diversos autores han abordado las dificultades que representa para la comprensión el lenguaje de los manuales escolares. Se señalan características como: las nominalizaciones con su función categorizante y su configuración polifónico-argumentativa (García, Hall \& Marín, 2005); las formas de referir en estos manuales, que presuponen conocimiento; la tendencia a extender la información a toda una clase de individuos; la condensación de la información; la presencia de la densidad léxica; y el uso de tecnicalizaciones, entre otros (González, 2010).

En el área específica de la enseñanza de la historia, se han adelantado diversos estudios como: el discurso científico pedagógico (Del Río, 2004; García, 2004; Vallejo, 2004); los recursos lingüísticos que proyectan ideologías (Oteíza, 2006, 2011; Moss, 2010; Atienza, 2011; Chamorro, Barletta \& Mizuno, 2013; Barletta \& Chamorro, 2015); la polarización entre la autoimagen positiva de 'nosotros' y la imagen negativa de los otros (Atienza, 2011); y la representación de las mujeres a través de la historia (Fernández, 2011). También hay estudios que caracterizan las diferencias entre los géneros históricos narrativos y los interpretativos mediante el uso de un lenguaje progresivamente más abstracto y metafórico, así como el valor del lenguaje para formar lectores críticos y dispuestos a la participación democrática (Giudice \& Moyano, 2011).

A nivel de terminología, los textos por lo general hacen algún esfuerzo por resaltar, identificar y hasta definir los términos que se prevé pueden ser difíciles para los alumnos (Mizuno, 2015). Los docentes, por lo general, están conscientes de las dificultades en el manejo de los términos nuevos, pero pocas veces son conscientes de la forma como la gramática de los libros de texto puede representar un obstáculo aún mayor para la comprensión de los fenómenos de la historia (Chamorro \& Moss, 2011).

Por otro lado, evidencias de diferentes contextos señalan que los niños y las niñas ingresan a la escuela con diferentes habilidades discursivas (Bernstein, 2003; Hasan, 2005). Por lo general, las clases dominantes manejan un mayor repertorio de registros, 
por lo cual están más preparadas para asimilar nuevos registros con mayor rapidez (Hasan, 1989; Painter, 1996). A esto se suma que los discursos que manejan guardan mayor semejanza con los discursos del ámbito académico que se maneja en las escuelas. Por otro lado, los trabajos de Bernstein (1975, 2003) y Williams (1999) entre otros, han mostrado cómo el discurso que se maneja en el aula es ajeno a las clases menos favorecidas.

Rose (2006), con base en sus investigaciones en escuelas australianas con estudiantes de educación primaria, secundaria y terciaria, insiste en que una de las formas en que se mantienen las desigualdades al término de la escuela radica en la poca o nula atención que prestan los maestros durante los años de la escuela secundaria a enseñar a aprender a partir de la lectura de los diferentes tipos de texto mediante los cuales se accede al conocimiento. Esto, si bien puede no afectar a los estudiantes más diestros, que de alguna manera aprenden a perfeccionar sus habilidades leyendo grandes cantidades de textos escritos que los preparan para el acceso a la universidad, deja a los otros ad portas del fracaso escolar, incapaces de comprender los discursos escritos, y con la única opción de dirigirse a las ocupaciones vocacionales o manuales (Rose, 2006).

Es por ello que varios investigadores subrayan la necesidad de estudiar cómo el conocimiento se construye en las diferentes áreas del saber, así como la urgencia de la enseñanza explícita del lenguaje de las mismas (Rose \& Acevedo, 2006). Al respecto, Bernstein (2003: 53) subraya el papel del libro de texto como "el medio pedagógico y la relación social fundamental".

En este sentido, en los últimos 20 años, hemos analizado, en detalle y de manera minuciosa, el lenguaje de 10 textos escolares en las áreas de Ciencias Sociales y Ciencias Naturales. Estos análisis nos han permitido caracterizar, a nivel global, el registro de los textos y su organización de la información; a nivel de las metafunciones ideacional, interpersonal y textual. Además, el análisis de los textos ha ido de la mano con la observación de la interacción maestro-texto-alumno que se genera en el aula alrededor de su contenido. Esto nos ha permitido identificar rasgos discursivos que, con una adecuada mediación de parte del docente, pueden facilitar la comprensión y el aprendizaje.

En este artículo analizamos los resultados de una investigación ${ }^{1}$ que pretendía determinar en qué medida el lenguaje de dos textos escolares de octavo grado de Ciencias Sociales había cambiado su manera de presentar la información. El capítulo analizado trata sobre la Revolución Industrial con el propósito de examinar el uso de los verbos del tipo ser, estar y tener, que aquí llamaremos procesos relacionales de acuerdo con la teoría de la Lingüística Sistémico-Funcional (Halliday, 2004a). Mostraremos cómo ellos contribuyen no solo a distanciar a los lectores de los fenómenos estudiados, sino también a presentar una visión entumecida de la historia. 
Inicialmente describiremos la perspectiva sistémico-funcional de lenguaje y las categorías utilizadas para el análisis del texto. Posteriormente, presentaremos el análisis de los procesos relacionales encontrados para discutir luego su impacto en la comprensión de los lectores estudiantes.

\section{Marco teórico}

\subsection{Representación del mundo a través del lenguaje}

La gramática sistémico-funcional (Halliday, 2004a) establece tres funciones principales de la comunicación lingüística llamadas 'metafunciones' del lenguaje: la ideacional, que se refiere a la representación por medio del lenguaje de la realidad tanto interna como externa, tanto social como natural; la interpersonal, que realiza las relaciones entre interlocutores; y la textual, que organiza la información dentro del mensaje.

En este artículo, haremos referencia primordialmente a la primera; la ideacional, ya que nuestro tema es la representación del mundo que se evidencia en un texto escolar de Ciencias Sociales. La metafunción ideacional es realizada a nivel gramatical principalmente por medio del sistema de la transitividad, la cual se describe a continuación.

\subsubsection{La transitividad}

Dentro del análisis de la metafunción ideacional, lo que nos concierne aquí es el sistema que utilizamos para representar los patrones de nuestra experiencia $y$, por ende, imponerle a la infinita variedad y vaivén de los eventos, cierto orden. En lingüística sistémico-funcional, esto se llama el sistema de la transitividad (Halliday, 2004a). Este sistema se basa en el principio general de que la realidad está conformada por procesos de diferentes tipos: suceder, actuar, sentir, pensar, ser, estar y tener (Halliday, 2004a).

Los tres tipos principales de procesos se distribuyen de la siguiente manera: la experiencia externa: procesos materiales (suceder y actuar); la experiencia interna: procesos mentales (sentir y pensar); y la clasificación e identificación: procesos relacionales (ser, estar y tener).

Otros tipos menos frecuentes de proceso son: la manifestación externa de funciones internas: los procesos conductuales (por ejemplo, llorar, sonreír); la representación lingüística de relaciones simbólicas: los procesos verbales (por ejemplo, decir, significar); y la existencia de un fenómeno: los procesos existenciales (hay, existen) (Halliday, 2004a). 
Al narrar hechos ocurridos en el pasado, lo más natural es utilizar principalmente procesos materiales que describan acciones y sucesos. De hecho, en los textos narrativos, suele ser así: los hechos se describen mediante procesos materiales y sólo aparecen procesos relacionales en pasajes descriptivos que especifican características de personajes, lugares u objetos. Sin embargo, en los textos académicos en general, y específicamente en los textos escolares, encontramos el uso frecuente de procesos relacionales para describir hechos y acciones. A continuación, veremos cómo funciona esto y cuáles son algunas de sus implicaciones.

\subsubsection{Los procesos relacionales}

Existen dos clases principales de proceso relacional con funciones diferentes: el proceso relacional atributivo que asigna un fenómeno a una clase o describe una característica del mismo; y el proceso relacional identificador que le asigna una identidad o definición a un fenómeno (Halliday, 2004a). A continuación veamos con más detenimiento estos tipos de procesos.

a) E1 proceso relacional atributivo. Este tipo de proceso se utiliza primordialmente en la descripción de personas, objetos, eventos, abstracciones, o procesos o propiedades nominalizados (ver sección 1.2). A estos fenómenos se les asigna una característica, una posesión o una circunstancia (localización, extensión, causa, manera, papel, acompañamiento, contingencia, asunto, punto de vista) (Halliday, 2004a). En el análisis, al fenómeno descrito se le llama 'Portador' y a la característica, posesión o circunstancia, se le llama 'Atributo'.

(1) El siglo XIX fue un período muy importante, tanto en el ascenso de los Estados Unidos como potencia, como en la formación de Canadá, hoy día no menos poderosa industrialmente. (CS: 120) ${ }^{2}$

(2) Estas nuevas relaciones hicieron que los beduinos, que tenían una sola lengua, intentaran salir del aislamiento en busca de unidad nacional. (M7: 60)

(3) En Santiago de Chile los criollos más opulentos instalaron la junta, el 18 de septiembre, bajo la presidencia del capitán general, pero duró poco tiempo. (C: 27)

El análisis transitivo de estos tres ejemplos se resume en la Tabla 1.

Tabla 1. Proceso relacional atributivo.

\begin{tabular}{|l|l|l|}
\hline Portador & Proceso & \multicolumn{1}{c|}{ Atributo } \\
\hline $\begin{array}{l}\text { El siglo } \\
\text { XIX }\end{array}$ & fue & $\begin{array}{l}\text { muy importante, tanto en el ascenso de los Estados Unidos como } \\
\text { potencia, como en la formación de Canadá, hoy día no menos } \\
\text { poderosa industrialmente (característica) }\end{array}$ \\
\hline $\begin{array}{l}\text { los } \\
\text { beduinos }\end{array}$ & tenían & una sola lengua (posesión) \\
\hline la junta & duró & poco tiempo (circunstancia de extensión temporal) \\
\hline
\end{tabular}


En algunos casos, encontramos que el atributo no se expresa de forma independiente sino que está integrado en el sentido del proceso, lo que Halliday (2004a: 222) denomina "proceso cualitativo". En (4) y en la Tabla 2, se presenta un ejemplo analizado de un proceso relacional cualitativo.

(4) Con la Ley de Cercamientos de 1727, se legalizaron estas apropiaciones. (H:13)

Tabla 2. Proceso relacional cualitativo.

\begin{tabular}{|l|l|c|}
\hline $\begin{array}{c}\text { Portador } \\
\text { (proceso + atributo) }\end{array}$ & $\begin{array}{l}\text { Proceso cualitativo } \\
\text { (proceso + atributo) }\end{array}$ & \multicolumn{1}{c|}{ Circunstancia } \\
\hline Estas apropiaciones & $\begin{array}{l}\text { se legalizaron } \\
\text { (se volvieron legales) }\end{array}$ & Con la Ley de Cercamientos de 1727 \\
\hline
\end{tabular}

Con mucha frecuencia, cuando el proceso relacional atributivo es de tipo cualitativo, viene asociado con un tercer participante, conocido como 'Atribuidor' (Halliday, 2004a); es decir, la entidad que asigna la relación de atribución, como se ve en el ejemplo (5):

(5) Este período se basó en la industria del algodón, la cual creció y dinamizó toda la economía británica. (H: 16)

En este caso, 'la industria del algodón' le otorga a la economía británica la característica de dinámica. La relación establecida por el proceso relacional es entre la economía británica y el atributo 'dinámica'; la industria del algodón es la que crea las condiciones para que se dé dicha relación. Esta figura se resume en la Tabla 3.

Tabla 3. Proceso relacional con Atribuidor.

\begin{tabular}{|c|c|c|}
\hline $\begin{array}{c}\text { Proceso Portador } \\
\text { (cualitativo) }\end{array}$ & $\begin{array}{c}\text { Atributo } \\
\text { (contenido en el proceso) }\end{array}$ & Atribuidor \\
\hline Dinamizó la economía británica & [dinámica] & la industria del algodón \\
\hline
\end{tabular}

b) El proceso relacional identificador. Este tipo de proceso sirve para identificar o definir fenómenos particulares. Indica una equivalencia entre las dos partes de una relación. En los textos escolares, se utiliza sobre todo para definir términos, especificar funciones y establecer taxonomías:

(6) ¿'Tienes idea de quiénes eran los agresores y quiénes los agredidos? (Identificación) (CS: 120)

(7) Para el siglo XVIII, Gran Bretaña comprendía los reinos de Inglaterra, Irlanda y Escocia que se unió en 1707. (Definición por enumeración de partes) (H: 12) 
(8) Lo sucedido en Santafé de Bogotá en 1810, no fue un hecho aislado, constituyó la culminación de movimientos juntistas previos en Cartagena, Socorro, Cali y Pamplona. (Identificación) (C: 26)

Ambos tipos de proceso relacional son frecuentes en los textos escolares de ciencias, pero nuestros análisis sugieren que en Ciencias Sociales suelen predominar los atributivos mientras que en Ciencias Naturales, donde prima la intención de establecer taxonomías, son más frecuentes los identificadores (Moss, Mizuno, Ávila, Barletta, Carreño, Chamorro \& Tapia, 2003; Moss, 2013). Por este motivo, en este artículo, centraremos nuestra atención en los procesos relacionales atributivos.

En la elaboración de un texto, la escogencia de procesos relacionales para describir los hechos de la experiencia tiene ciertas implicaciones con respecto a la visión del mundo que se presenta; se refleja la experiencia en términos de 'ser' o 'estar' en vez de 'hacer' o 'sentir'. Es decir, se construye el proceso de cambio de manera estática, sin insumo de energía y sin fases definidas (Halliday, 2004a). Por consiguiente, el uso de una alta proporción de procesos relacionales en un texto que describe eventos y procesos históricos, construye una visión de la historia en la que dichos eventos y procesos no ocurren sino que se describen como hechos ya cumplidos a los cuales se les asignan ciertas características $y / o$ con los cuales se relacionan ciertas circunstancias. Por otra parte, tanto atribuir características a los fenómenos como establecer relaciones lógicas entre ellos, son actos de interpretación. De esta manera, un texto de historia con un alto porcentaje de procesos relacionales necesariamente ofrece no sólo información acerca de los hechos sino una interpretación de los mismos (Wignell, 1998). A esto se aúna el hecho de que, al no explicitar que se trata de una interpretación, se tiende a crear la impresión de que se trata de la representación de la verdad. A continuación, explicaremos otro aspecto de la transitividad que, con frecuencia, co-ocurre con el uso de los procesos relacionales.

\subsection{Una dificultad del discurso escrito: La metáfora gramatical}

El término metáfora gramatical se refiere al fenómeno en que un significado que sería representado de manera congruente por una forma gramatical es representado de manera metafórica por otra forma gramatical. La forma congruente es una manera de expresar el significado intuitivamente más cercano a los eventos del mundo externo. Tal es el caso de los procesos, los cuales se expresan de manera congruente mediante verbos, y metafóricamente mediante sustantivos. Esto no significa que la realización metafórica sea mejor o peor, o más o menos frecuente; sin embargo, posee algunas características particulares (Halliday \& Martin, 1993; González, 2004; Halliday, 2004b; Schleppegrell, Achugar \& Oteíza, 2004; Moss, 2013; Moss, Barletta, Chamorro \& Mizuno, 2013) que veremos a continuación. 
En el discurso científico, gran parte de las metáforas gramaticales son nominalizaciones: la expresión de procesos y propiedades en forma de sustantivos, en vez de verbos y adjetivos.

Por ejemplo, en (9), encontramos que los procesos 'formar', 'consolidar', 'desarrollar', 'difundir' e 'industrializar' son todos realizados por medio de sustantivos (nominalizaciones).

(9) En esta fase se desarrollaron las llamadas industrias pesadas: carbón, hierro

y acero. Estas industrias permitieron la formación, la consolidación, el desarrollo y la difusión de la industrialización. (H: 17)

A nivel conceptual, la nominalización masiva, característica del discurso científico "mantiene inmóvil a la realidad para permitir la observación y experimentación" (Halliday \& Martin, 1993: 15). Los procesos y las propiedades se objetivizan, y se representa un mundo en el que los objetos predominan y los procesos sirven simplemente para definir y clasificar. Un proceso o evento objetivizado, se presenta como un hecho acabado, incambiable, que no puede ser cuestionado ni refutado sino que debe ser aceptado por el lector como la realidad. Igualmente al desaparecer el sujeto del verbo, se pierde la asignación de responsabilidad. Así, en (9), no sabemos quién formó, consolidó, desarrolló, difundió e industrializó. La nominalización se presenta, no como creación de una conciencia humana, sino simplemente como entidad (Moss et al., 2003).

Otra clase de metáfora gramatical consiste en el uso de verbos causales para establecer relaciones de causa-consecuencia. La forma congruente de expresar este tipo de relaciones es por medio del uso de conjunciones de causalidad para relacionar dos cláusulas, una de las cuales expresa el evento o fenómeno que constituye la causa y la otra el evento o fenómeno que constituye la consecuencia. Por ejemplo, la oración que se presenta en (10) y se analiza en la Tabla 4.

(10) Aparecieron las máquinas y por consiguiente se rompieron las tradicionales formas de producir las cosas.

Tabla 4. Relación causa-efecto mediante conjunción.

\begin{tabular}{|l|l|l|}
\hline \multicolumn{1}{|c|}{$\begin{array}{c}\text { Causa } \\
\text { Proceso material }\end{array}$} & \multicolumn{1}{c|}{$\begin{array}{c}\text { Conjunción } \\
\text { causal }\end{array}$} & \multicolumn{1}{c|}{$\begin{array}{c}\text { Consecuencia } \\
\text { Proceso material }\end{array}$} \\
\hline $\begin{array}{l}\text { Aparecieron las } \\
\text { máquinas }\end{array}$ & $\begin{array}{l}\text { y por } \\
\text { consiguiente }\end{array}$ & $\begin{array}{l}\text { se rompieron las tradicionales formas de producir } \\
\text { (cosas). }\end{array}$ \\
\hline
\end{tabular}

En el discurso científico frecuentemente se simplifica gramaticalmente este tipo de complejo de cláusulas por medio de nominalizar los eventos que son causa y consecuencia y relacionarlos por medio de un verbo causal, como por ejemplo, 'provocar', 'producir', 'causar'. De esta manera, el complejo de dos cláusulas se reduce 
a una cláusula al interior de la cual se realizan la causa y la consecuencia como participantes. El ejemplo (10a) y la Tabla 5 muestran la versión metafórica del ejemplo (10).

(10a) La aparición de las máquinas significó una gran ruptura con las tradicionales formas de producción. (H: 15)

Tabla 5. Relaciones causa-efecto mediante procesos relacionales.

\begin{tabular}{|l|l|l|}
\hline \multicolumn{1}{|c|}{$\begin{array}{c}\text { Causa } \\
\text { Nominalización }\end{array}$} & \multicolumn{1}{c|}{$\begin{array}{c}\text { Verbo } \\
\text { causal }\end{array}$} & \multicolumn{1}{c|}{ Nominalización } \\
\hline $\begin{array}{l}\text { La aparición de las } \\
\text { máquinas }\end{array}$ & significó & $\begin{array}{l}\text { una gran ruptura con las tradicionales formas de producción } \\
\text { (H. 15.1) }\end{array}$ \\
\hline
\end{tabular}

El proceso de metaforización lleva al desarrollo de lo que Halliday (2004b) llama 'la cláusula preferida de la ciencia’ que consiste en dos nominalizaciones conectadas por un proceso relacional. Es decir, los eventos narrados son realizados gramaticalmente por sustantivos y el verbo, en vez de describir una acción o evento, simplemente establece una relación entre estas entidades. A continuación, describiremos el papel de este lenguaje metafórico en el desarrollo ontológico del lenguaje, aspecto que los docentes deben tener en cuenta cuando trabajan un texto con los estudiantes.

\subsection{El desarrollo del lenguaje de la ciencia}

En sus estudios del lenguaje de las ciencias, Halliday (2004b) ha identificado una serie de etapas de progresiva abstracción y generalización en el desarrollo de las lenguas, que iniciaron con un lenguaje que probablemente servía para significar las entidades concretas; luego, seguiría la 'generalización', del nombre propio al nombre común, que haría posible la existencia del denominado 'sentido común', también llamado 'conocimiento cotidiano'; en un nivel mayor de evolución del lenguaje, encontramos la 'abstracción' en la que se pasa de categorizaciones concretas a otras más abstractas, de manera que se re-teoriza el sentido común: son los inicios de la ciencia formal, es decir, el lenguaje escrito. Por último se llegaría a la etapa de la 'metáfora gramatical' que se caracteriza por establecer relaciones entre conceptos y producir una nueva re-teorización del conocimiento, estableciendo el conocimiento científico y técnico con sus correspondientes discursos.

De manera parecida, Halliday (2004b) ha identificado también una serie de etapas por las que pasa el aprendiz. La primera etapa sería la del protolenguaje, utilizado únicamente para la comunicación con los seres más allegados. La siguiente etapa está caracterizada por la generalización, representada por el manejo del lenguaje oral de la lengua materna. La etapa posterior se caracteriza por su nivel de abstracción y corresponde a la lengua escrita. Finalmente, se llega al estilo científico caracterizado por altos niveles de abstracción y generalización, expresados por medio de la metáfora 
gramatical, especialmente la nominalización compleja. Así como afirma Vygotsky (1986), se parte de la experiencia directa y, en etapas posteriores, el individuo se desprende cada vez más de la experiencia sensible e ingresa al terreno de la conceptualización. Los textos didácticos, entonces, deberían empezar, a nivel de primaria, con un discurso muy concreto y contextualizado para volverse, a lo largo de la educación secundaria, progresivamente, más abstractos y más metafóricos hasta llegar al tipo de cláusula preferida por la ciencia.

\section{Metodología}

Para fines de este artículo, hemos escogido un capítulo del texto más reciente de nuestro corpus, 'Hipertexto Sociales 8' de Santillana publicado en 2010. El libro es una obra colectiva concebida, diseñada y creada por el Departamento Editorial de Santillana S. A., en Bogotá, Colombia, cuyos autores son 6 profesores de la Universidad Nacional de Colombia. Es distribuido por medio de las librerías comerciales del país y era en su momento uno de los más usados en los colegios oficiales de la región. Trabajamos aquí con un solo capítulo de un solo libro con el propósito de examinar detenidamente la forma como el texto construye su descripción de un proceso histórico específico: la Revolución Industrial. Este tema es central en el currículo de octavo grado de Ciencias Sociales en Colombia (MEN, 2004) y como tal, ha sido un objeto de estudio contrastivo a través de nuestras investigaciones. Nuestros análisis nos muestran que, a través de los años, las escogencias lingüísticas de los textos escolares para representar la historia son recurrentes (Moss et al., 2003; Moss, Natale \& Oteíza, 2009; Barletta \& Chamorro, 2015).

El lenguaje de este capítulo es analizado desde la perspectiva sistémico.-funcional; específicamente nos centramos en la realización de los procesos y participantes de las cláusulas relacionales que pueden afectar los procesos de enseñanza y aprendizaje de la historia: los procesos relacionales cualitativos; los Atribuidores como participantes de las cláusulas relacionales; las metáforas léxicas y la expresión metafórica de la causalidad mediante procesos relacionales, los participantes abstractos y las nominalizaciones en las cláusulas relacionales que hacen referencia a pasajes largos de texto. También tenemos en cuenta el efecto creado por párrafos o grupos de párrafos que combinan varias de estas características.

Para la identificación y clasificación de los procesos analizamos una a una las cláusulas que configuran el texto. Según la LSF, una cláusula "es una estructura de palabras y grupos de palabras que construyen una actividad que involucra personas y cosas" (Martin \& Rose, 2007). La actividad es constituida por procesos que se realizan mediante verbos. Las personas y cosas funcionan como participantes en la cláusula. En la cláusula también puede haber cualidades, momentos, lugares u otros elementos circunstanciales. 
El tipo de Proceso (verbos) y el tipo de Participante (personas o cosas) definen la configuración del mundo tanto social como natural y como tales son los elementos nucleares de la cláusula. Por su parte, los elementos circunstanciales (Circunstancias) tienden a ser más periféricos y pueden estar incluidos o no.

Nuestro análisis nos permitió establecer que este capítulo configura la realidad mediante 70 procesos relacionales y 150 materiales.

El libro escogido va dirigido a estudiantes de educación básica en Colombia que cursan octavo grado, los cuales oscilan entre 13 y 15 años de edad. El libro pretende seguir los lineamientos establecidos por el Ministerio de Educación Nacional. El capítulo que escogimos para estudio fue la Unidad 1 titulada 'La Revolución industrial', la cual está dividida en seis secciones así: la primera, La Revolución industrial, en la cual se presenta la definición de revolución, la definición de Revolución industrial y las causas que la produjeron; la segunda, Contexto geográfico, en la cual se hace una ubicación temporal y geográfica del hecho histórico; la tercera, Revoluciones burguesas, Cambios en la propiedad de la tierra, Revolución agrícola, y la Revolución demográfica; la cuarta, los Fundamentos de la Revolución industrial, que se encuentra dividida en tres: El mercado interno, El mercado externo, y El gobierno; la quinta, Principales cambios en la revolución industrial, se subdivide en tres: Avances tecnológicos, Nueva organización del trabajo, Urbanización; la sexta, Fases de la Revolución industrial, dividida en Primera fase y Segunda fase; y por último, La Revolución industrial en otros países, dividida así: Bélgica, Francia, Alemania y Estados Unidos.

En los ladillos encontramos varias secciones, distribuidas a lo largo del capítulo, tituladas: Ubícate en la historia, Para pensar..., Para responder..., Vocabulario de términos, Para saber más, ¿Sabías que...? Además, cada una de las páginas presenta fotografías o dibujos que pretenden ilustrar el tema tratado.

\section{Resultados}

\subsection{Procesos cualitativos}

Una de las características sobresalientes del texto es la abundancia de procesos cualitativos. De un total de 70 procesos relacionales, $31(44,6 \%)$ tienen esta característica, es decir, el proceso subsume la relación y el atributo, el cual intensifica. El párrafo que reproducimos abajo (11) ejemplifica este rasgo del lenguaje del texto, porque contiene tres de estos procesos.

(11) Gracias a su poder naval, Gran Bretaña consolidó un vasto imperio que le aseguró el suministro de materias primas, y el monopolio sobre amplios mercados coloniales. [...]. El comercio colonial intensificó la esclavitud, desde 
los proveedores de esclavos y de productos para su manutención, hasta su explotación en tareas agrícolas y mineras en las colonias. (H: 14)

El siguiente es el análisis de las cláusulas que contienen procesos relacionales atributivos

Tabla 6. Procesos relacionales atributivos.

\begin{tabular}{|l|l|l|}
\hline \multicolumn{1}{|c|}{ Proceso-atributo } & \multicolumn{1}{c|}{ Portador } & \multicolumn{1}{c|}{ Atribuidor } \\
\hline consolidó & un vasto imperio que... & Gran Bretaña \\
\hline aseguró & el suministro de materias primas & un vasto imperio \\
\hline intensificó & la esclavitud ... & el comercio colonial \\
\hline
\end{tabular}

En esta representación, la Revolución Industrial consiste en un proceso mediante el cual los fenómenos existentes en Gran Bretaña adquieren nuevas cualidades o intensifican las que ya poseen con lo cual la Revolución prácticamente se reduce a un proceso de evolución.

\subsection{Los Atribuidores}

Una de las formas de presentar la información utilizando procesos relacionales atributivos es que se presenta la información indicando que hay un elemento que concede el atributo, al que hemos llamado Atribuidor. En el capítulo analizado encontramos un total de 18 y están expresados así: 11 abstracciones, 3 objetos, 2 instituciones, 1 complejo de nominalizaciones, 1 generalización. Analicemos el siguiente caso, en donde al Atribuidor es una institución.

(12)... en el continente persistían estructuras feudales como la servidumbre, los gremios, las aduanas y tributos señoriales, que dificultaron la producción, el comercio y la creación de mercados nacionales. (H: 18)

Tabla 7. Institución como Atribuidor.

\begin{tabular}{|l|l|l|}
\hline \multicolumn{1}{|c|}{ Atribuidor } & \multicolumn{1}{|c|}{$\begin{array}{c}\text { Proceso- } \\
\text { atributo }\end{array}$} & \multicolumn{1}{c|}{ Portador } \\
\hline $\begin{array}{l}\text { Estructuras feudales como la servidumbre, } \\
\text { los gremios, las aduanas y tributos } \\
\text { señoriales }\end{array}$ & $\begin{array}{l}\text { (que) } \\
\text { dificultaron }\end{array}$ & $\begin{array}{l}\text { la producción, el comercio y } \\
\text { creación de mercados } \\
\text { nacionales }\end{array}$ \\
\hline
\end{tabular}

En este caso, el Atribuidor es una institución (estructuras feudales) de la cual seguidamente se da ejemplos (la servidumbre, los gremios, las aduanas y los tributos señoriales) que son difíciles de comprender para una estudiante que por primera vez se enfrenta a este conocimiento, puesto que la mayoría de ellos dejaron de existir hace mucho tiempo en Europa y no se mencionan en el desarrollo de la historia de Colombia. Por consiguiente, es difícil que estos estudiantes puedan comprender por qué estas instituciones eran un obstáculo para el progreso. 
Veamos un caso de ente abstracto como Atribuidor:

(13) Los ferrocarriles consolidaron la producción de hierro y carbón, a la vez que ampliaron sus mercados externos, [...]. (H: 17)

Tabla 8. Ente abstracto como Atribuidor.

\begin{tabular}{|c|l|l|}
\hline Atribuidor & \multicolumn{1}{|c|}{ Proceso } & \multicolumn{1}{c|}{ Portador } \\
\hline \multirow{2}{*}{ Los ferrocarriles } & consolidaron & la producción de hierro y del carbón \\
\cline { 2 - 3 } & Ampliaron & los mercados externos \\
\hline
\end{tabular}

Como podemos apreciar, en este caso, el Atribuidor es la institución 'ferrocarriles', la cual es un ente abstracto que esconde las personas que manejan esta institución. Estas personas podrían ser empresarios, ministros, obreros. De la manera como plantea el texto los hechos de la historia, las personas no son las que participan en el proceso de consolidar la producción y ampliar los mercados, sino que estas industrias se manejan solas y progresan sin el apoyo de nadie.

Lo importante de mencionar a personas como Atribuidores es que les permite a los estudiantes tener una visión concreta de la historia que sería más fácil de entender puesto que tienen una edad (12-13 años) en la cual todavía están en el proceso de pasar de un pensamiento en concreto a un pensamiento abstracto. De acuerdo con Halliday (2004b: 29-30):

"la capacidad de comprender un lenguaje netamente abstracto se desarrolla gradualmente a lo largo de la adolescencia y la vida escolar; a los 12 años aún se necesitan componentes lingüísticos concretos para lograr una buena comprensión".

Otra ventaja de la inclusión de personas como Atribuidores sería que la completud en la información les permitiría a los estudiantes tener una visión más amplia de los hechos históricos.

\subsection{Las metáforas léxicas}

Otro rasgo interesante del texto lo constituyen los procesos relacionales que son a su vez metáforas léxicas. Los ejemplos (14) y (15), presentados en la Tabla 9, ilustran este aspecto.

(14) La sociedad británica experimentó una serie de transformaciones económicas, sociales y culturales (H: 11).

(15) La industria del hierro disfrutó una serie de mejoras técnicas (H: 17) 
Tabla 9. Procesos relacionales como metáforas léxicas.

\begin{tabular}{|l|l|l|}
\hline \multicolumn{1}{|c|}{ Portador } & \multicolumn{1}{c|}{ Proceso } & \multicolumn{1}{c|}{ Atributo } \\
\hline $\begin{array}{l}\text { la sociedad } \\
\text { británica }\end{array}$ & experimentó & $\begin{array}{l}\text { una serie de transformaciones económicas, sociales y } \\
\text { culturales }\end{array}$ \\
\hline $\begin{array}{l}\text { La industria del } \\
\text { hierro }\end{array}$ & disfrutó & una serie de mejoras técnicas \\
\hline
\end{tabular}

En virtud de la expresión metafórica 'experimentó', cuyo significado literal es “tener experiencia sensorial de algo" (Seco \& Ramos, 1999: 2075), en el ejemplo "una serie de transformaciones económicas y sociales" se percibe como un fenómeno externo a la sociedad misma con el cual ella tiene que convivir.

En el ejemplo (15), la escogencia del proceso relacional 'disfrutó' le confiere al Portador, 'la industria del hierro', cualidades en el ámbito del sentir, típicas de los entes con consciencia, lo cual contrasta con el carácter abstracto, intangible, y despersonalizado de esa entidad.

\subsection{Los verbos causales}

Como se ha mencionado en la sección 1.2, los procesos relacionales atributivos a veces son utilizados en el contexto de la metaforización gramatical de la causalidad. En estos casos, el proceso establece una relación de tipo causal entre un fenómeno atribuidor y el hecho de la atribución; es decir, el Atribuidor aparece como causa de la relación entre el Portador y su Atributo. Además, típicamente se trata de un proceso cualitativo (sección 'Los procesos relacionales atributivos') que subsume la relación y el atributo. Veamos un ejemplo:

(16) [El petróleo] permitió el posterior surgimiento de industrias como el plástico y los productos sintéticos, así como del automóvil. (H: 19)

Aquí vemos cómo el Atribuidor, 'el petróleo', aparece como el responsable de la relación entre el Portador, 'el posterior surgimiento de industrias como el plástico y los productos sintéticos, así como del automóvil', y el Atributo, 'posible', que está implícito en el proceso, 'permitió'. Este análisis se presenta en la Tabla 10.

Tabla 10. Proceso cualitativo causal.

\begin{tabular}{|l|l|l|}
\hline Atribuidor & \multicolumn{1}{|c|}{$\begin{array}{c}\text { Proceso- } \\
\text { atributo }\end{array}$} & \multicolumn{1}{c|}{ Portador } \\
\hline $\begin{array}{l}{[\text { El }} \\
\text { petróleo] }\end{array}$ & Permitió & el posterior surgimiento de industrias como \\
\hline & (hizo posible) & $\begin{array}{l}\text { el plástico y los productos sintéticos, así como del } \\
\text { automóvil }\end{array}$ \\
\hline
\end{tabular}

Esta configuración gramatical, con uso del proceso cualitativo causal, tiene el efecto de 'englobar' en el concepto 'el petróleo' toda una serie de procesos y de 
acciones humanas: descubrimiento de yacimientos de petróleo, desarrollo de la tecnología para explotarlos y usar el producto en una variedad de aplicaciones. El hidrocarburo está presentado como responsable de todo este complejo de eventos y acciones.

\subsection{Participantes abstractos o nominalizados}

La ocurrencia de Atributos realizados como participantes abstractos o nominalizados es otro de los rasgos identificados en el texto, como se puede apreciar en el ejemplo (17):

(17) La revolución industrial fue un proceso que tuvo su origen en Gran Bretaña a mediados del siglo XVIII y que consistió en un acelerado crecimiento económico, acompañado de grandes transformaciones sociales y tecnológicas, [...]. Por primera vez, una sociedad superó los límites de la economía agraria e inició una constante y rápida producción masiva de manufacturas a un bajo costo, obteniendo grandes beneficios económicos. (H: 12)

Tabla 11. Participantes abstractos.

\begin{tabular}{|l|l|l|}
\hline \multicolumn{1}{|c|}{ Portador } & Proceso & \multicolumn{1}{c|}{ Atributo } \\
\hline La revolución industrial & Tuvo & origen en Gran Bretaña a mediados del siglo XVIII \\
\hline Una sociedad & Superó & los límites de la economía agraria \\
\hline
\end{tabular}

El párrafo citado en el ejemplo (17) se encuentra en la sección de introducción a la unidad donde se presentan los conceptos que se van a tratar. En la primera oración, definen un concepto específico que es 'Revolución industrial'. Para ello se utilizan características y atributos expresados con nominalizaciones (acelerado crecimiento económico, grandes transformaciones sociales y tecnológicas, cambios en el sistema de producción). Después, en la segunda oración, se menciona un hecho a través de la relación del Portador (sociedad) que es una abstracción con un Atributo expresado a través de una metáfora (límites de la economía agraria), elementos estos difíciles de entender para un lector inexperto. Analicemos el siguiente caso:

(18) El proceso de industrialización británico se apoyó en un mercado interior bastante desarrollado, una posición hegemónica en el mercado mundial y el respaldo del gobierno.(H: 14)

Tabla 12. Complejo de nominalizaciones como portador.

\begin{tabular}{|l|l|l|}
\hline \multicolumn{1}{|c|}{ Portador } & Proceso & \multicolumn{1}{c|}{ Atributo } \\
\hline El proceso de & $\begin{array}{l}\text { se } \\
\text { apoyó }\end{array}$ & en un mercado interior bastante \\
\hline $\begin{array}{l}\text { Industrialización } \\
\text { británico }\end{array}$ & & $\begin{array}{l}\text { desarrollado, una posición hegemónica en el mercado } \\
\text { mundial y el respaldo del gobierno }\end{array}$ \\
\hline
\end{tabular}


El párrafo presentado en el ejemplo [18] y analizado en la Tabla 12 solo cuenta con una oración y es el inicio de una sección titulada 'Fundamentos de la Revolución Industrial'.

En ambos párrafos el texto presenta a los portadores en términos abstractos, como una abstracción (en el primer caso) y como un conjunto de nominalizaciones lo cual da como resultado un complejo de nominalizaciones (el proceso de industrialización segundo caso-). Esta manera de presentar la historia muestra los hechos como cosas que no afectan a nadie y que nadie ha sido el causante de ellos. Sin embargo, tanto la 'Revolución industrial' como el 'Proceso de industrialización' son hechos históricos y como tales han sido vividos, causados y han afectado a las personas, pero en estos dos ejemplos no se muestran de esa manera. Esto evita un compromiso con una posición ideológica o con los intereses de algún grupo social. Tampoco propone las diferentes visiones posibles para abordar este proceso histórico y da muestras de una ideología que evita responsabilidades con la sociedad.

\subsection{Las nominalizaciones generalizadoras}

Arriba nos hemos referido varias veces a la dificultad que puede representar para lectores no expertos el enfrentarse con un texto de historia construido no con referencia a acciones concretas de seres humanos sino con referencia a generalizaciones y abstracciones que se relacionan unas con otras de manera también abstracta. Hemos notado que el uso frecuente de los procesos relacionales suele suponer la nominalización de los participantes, aumentando así los niveles de abstracción del texto. A estas dificultades se suma el hecho de que, en ocasiones, el Portador de una cláusula relacional atributiva es una nominalización generalizadora que hace referencia a un pasaje largo de texto precedente cuyo contenido el lector debe poder mantener en su memoria de trabajo para poder comprender la relación que se está estableciendo. Ahora bien, este uso de la nominalización en lugar inicial de la cláusula realiza una importante función estructuradora dentro del discurso (Halliday \& Martin, 1993; Moss et al., 2003) y cuando el pasaje de texto al cual se hace referencia no es muy largo, puede constituirse en un apoyo para el lector. Sin embargo, cuando el pasaje referido es excesivamente largo, es probable que ocurra lo contario; que la exigencia para la memoria de trabajo sea tal que la referencia termina dificultando la comprensión. En el ejemplo [19], el Portador, 'La Revolución industrial' hace referencia a toda la información que se ha dado hasta el momento en la unidad: cinco páginas.

[19] La Revolución industrial se divide en dos grandes fases comprendidas entre 1780 y 1895 . (H: 16)

De esta manera, una oración aparentemente sencilla y que los estudiantes tal vez tengan la impresión de comprender, puede resultar en realidad sin sentido para ellos, 
debido a que no están en capacidad de darle un significado profundo y complejo a la expresión 'La Revolución industrial' (Chamorro, Mizuno \& Moss, 2003).

Otro ejemplo de este fenómeno lo encontramos en [20] en el que el Portador, 'Los avances industriales británicos' también se refiere a todo lo expresado hasta el momento en la unidad, que para este momento ya son siete páginas.

(20) Los avances industriales británicos se expandieron sobre Europa y Norteamérica (...). (H: 18)

\subsection{Párrafos con altos niveles de abstracción}

En el marco de este texto, existen algunos párrafos que reúnen gran número de las características arriba descritas y que, por lo tanto, presentan niveles muy altos de abstracción. El párrafo que analizaremos a continuación es ejemplo de este fenómeno:

(21) Este nuevo sistema de producción industrial, apoyado en un constante progreso tecnológico, consolidó un mercado a nivel mundial y generó una división internacional del trabajo, gracias a lo cual unos países se especializaron en productos industriales y otros en materias primas. Este proceso permitió el surgimiento y desarrollo de un nuevo sistema económico: el capitalismo. $(\mathrm{H}$ : 11)

Este párrafo contiene tres procesos relacionales atributivos: 'consolidó', 'se especializaron' y 'permitió'. Los tres son procesos cualitativos, es decir, subsumen proceso y atributo; el tercero, 'permitió', es además verbo causal. Estos procesos cualitativos son asociados con dos participantes Atribuidores. El primero, 'este nuevo sistema de producción industrial, apoyado en un constante progreso tecnológico’ es lo que hemos denominado un complejo de nominalizaciones; con esto queremos decir que el participante es realizado por dos (o más) grupos nominales cada uno de los cuales es o contiene una nominalización; en este caso, 'este nuevo sistema de producción industrial' y '(apoyado en) un constante progreso'. El segundo Atribuidor es una nominalización generalizadora, 'este proceso' que hace referencia a todo lo contenido en la oración anterior. Al mismo tiempo, los Portadores, 'un mercado a nivel mundial', 'unos países' y 'otros (países)', también son abstracciones, de manera que todos los participantes del párrafo son abstractos.

El hecho de que la totalidad de los participantes sean abstractos y que los hechos se representen no en términos de acciones y eventos sino en términos de asignación de atributos, muestra el devenir histórico, no como un proceso cambiante que lo generan las personas para obtener beneficio y que trae consecuencias favorables o desfavorables para diferentes grupos sociales, sino como un proceso en que las personas no intervienen; los cambios se van generando solos porque así tiene que ser. Justamente, el sistema de producción industrial del siglo XVIII fue un hecho histórico 
de gran importancia por la participación que tuvieron los ciudadanos de todos los estratos sociales, de todas las edades y de ambos sexos, pero este hecho no se puede apreciar en el texto. Por otra parte, hechos como la división entre países especializados en productos industriales y otros especializados en producción de materias primas, que han tenido resultados trascendentales en la formación del mundo inequitativo que conocemos actualmente, se presentan como resultados inevitables del progreso tecnológico, desligados de los intereses de diversos grupos sociales y/o nacionales.

\section{CONCLUSIONES}

Un aspecto destacado del estudio, lo constituye la realización de los procesos relacionales. En el texto analizado, además de presentarse bajo la forma prototípica de 'ser', 'estar' o 'tener', encontramos los procesos relacionales cualitativos que subsumen en sí mismos la relación y el atributo, tales como consolidar, asegurar, intensificar, dificultar. Otras formas de manifestarse son las metáforas léxicas (como por ejemplo experimentar, disfrutar) y procesos que establecen una relación causal (permitir, significar). Otro aspecto notorio es la naturaleza abstracta de los participantes en las cláusulas relacionales: participantes abstractos (revolución, sociedad, proceso de industrialización) y nominalizaciones generalizadoras que recogen información presentada en párrafos o páginas previas (los avances industriales británicos).

La revolución industrial en el capítulo analizado se presenta como un nuevo estado de cosas, el cual se caracteriza y se interpreta. Sin embargo, el carácter abstracto y hermético de su representación en este texto vuelve este fenómeno prácticamente inescrutable para neófitos. Como lo diría Rose (1998: 264):

"en lugar del mundo percibido por los sentidos en el cual tienen lugar procesos en los cuales participan personas de verdad, cosas, lugares y cualidades, la realidad se percibe como una estructura generalizada de abstracciones".

A menos que los maestros sean conscientes de estas características del texto, una buena parte de sus lectores no podrán saltar sus altísimas murallas. No solo tendrán dificultad para comprender qué sucedió en el pasado, sino que también posiblemente se vean impedidos para negociar su lugar en la construcción y en la representación de la historia.

\section{REFERENCIAS BIBLIOGRÁFICAS}

Aguirre, J. (2015). El manual escolar como objeto de investigación en historia de la educación: Apuntes al texto de geografía. Historia y Sociedad, 28, 247-272.

Atienza, E. (2007). Discurso e ideología en los libros de texto de ciencias sociales, Discurso \& Sociedad, 1(4), 543-574. 
Atienza, E. (2011). La construcción de las identidades colectivas en los libros de Ciencias Sociales en España. En T. Oteíza \& D. Pinto (Eds.), En (re) construcción: Discurso, identidady nación en los manuales escolares de historia y de ciencias sociales (pp. 321-348). Santiago: Editorial Cuarto Propio.

Barletta, N. \& Chamorro, D. (2015). El texto escolar y el aprendizaje: Enredos y desenredos. Barranquilla: Ediciones Uninorte.

Beck, I., McKeown, M. G. \& Gromoll, E. W. (1989). Learning from social studies texts. Cognition and Instruction, 6, 99-158.

Bernstein, B. (1975). Class, codes and control. Towards a theory of educational transmission. Londres: Routledge.

Bernstein, B. (2003). Class, codes and control Vol. IV. The structuring of pedagogic discourse. Londres: Routledge.

Borja, J. \& Cordi, J. (1992). Ciencias Sociales 8: Educación básica secundaria. Bogotá: Santillana.

Caballero, B. Cote, J. Cristancho, H., Fajardo A., Maldonado C. \& Prieto, F. (2010). Hipertexto Sociales. Bogotá: Editorial Santillana.

Chamorro, D. \& Moss, G. (2011). La pedagogía de la simplificación: El estudio de la historia por medio de la pista y pesca. En T. Oteíza \& D. Pinto (Eds.), En (re) construcción: Discurso, identidad y nación en los manuales escolares de bistoria y de ciencias sociales (pp. 269-320). Santiago: Editorial Cuarto Propio.

Chamorro, D., Mizuno, J. \& Moss, G. (2003). Tergiversaciones y correspondencias: La metáfora y sus bemoles. Revista Latinoamericana del Discurso, 3, 29-47.

Chamorro, D., Barletta, N. \& Mizuno, J. (2013). El lenguaje para enseñar y aprender las ciencias naturales: Un caso de oportunidades perdidas para la formación ciudadana. Revista Signos. Estudios de Lingüistica, 46(81), 3-28.

Coffin, C. (2006). Historical discourse. The language of time, cause and evaluation. Londres: Continuum.

Del Río, H. (2004). Manuales de historia: Lectura comparada de dos textos. En P. Vallejo Llobet (Comp.), El discurso científico pedagógico: Aspectos de la textualización del saber enseñando (pp. 19-36). Bahía Blanca, Argentina: Editorial de la Universidad del Sur.

Eggins, S., Wignell, P. \& Martin, R. (1993). The discourse of history: Distancing the recoverable past. En M. Ghadessy (Ed.), Register analysis: Theory and practice (pp. 75-109). Londres: Pinter. 
Fernández, M. (2011). Derecho a sufragio femenino en Chile. Recontextualización discursiva del rol de las mujeres en los textos escolares de Historia y Ciencias Sociales. En T. Oteíza \& D. Pinto (Eds.), En (re) construcción: Discurso, identidady nación en los manuales escolares de historia y de ciencias sociales (pp. 349-383). Santiago: Editorial Cuarto Propio.

García, M. (2004). La construcción lingüística de la causalidad en el discurso científico. Ciencia de investigación y ciencia escolar. En P. Vallejo Llobet (Comp.), El discurso científico pedagógico: Aspectos de la textualización del saber enseñando (pp. $37-$ 50). Bahía Blanca, Argentina: Editorial de la Universidad del Sur.

García, N., Hall, B. \& Marín, M. (2005). Ambigüedad, abstracción y polifonía del discurso académico: Interpretación de las nominalizaciones. Revista Signos. Estudios de Lingüistica, 38(57), 49-60 [en línea]. Disponible en: http://dx.doi.org/10.4067/S0718-09342005000100004.

Giudice, J. \& Moyano, E. (2011). Género y formación de ciudadanos: La reconstrucción del período 1976-1983 en manuales argentinos para la escuela primaria. En T. Oteíza \& D. Pinto (Eds.), En (re) construcción: Discurso, identidad y nación en los manuales escolares de historia y de ciencias sociales (pp. 265-267). Santiago: Editorial Cuarto Propio.

González, N. (2004). La metáfora gramatical en los manuales de ciencia de nivel medio: Dificultades en su comprensión. En P. Vallejos. (Ed.), El discurso cientifico pedagógico: Aspectos de la textualización del saber enseñado (pp. 51-75). Bahía Blanca, Argentina: Editorial de la Universidad Nacional del Sur.

González, N. (2010). Las formas de referir y la información contextual en el discurso de los manuales escolares y las revistas de divulgación infantil. En V. Castel \& L. Cubo de Severino (Eds.), La renovación de la palabra en el bicentenario de la Argentina. Los colores de la mirada lingüística (pp. 587-596). Mendoza: Editorial FFyL, Universidad de Cuyo.

Halliday, M. A. K (2004a). An introduction to functional grammar. Londres: Arnold.

Halliday, M. A. K. (2004b). The language of science. Londres: Continuum.

Halliday, M.A.K. \& Martin, J. (1993). Writing science. Londres: The Falmer Press.

Hasan, R. (1989). Semantic variation and sociolintuistics. Australian Journal of Linguistics, 9(2), 221.

Hasan, R. (2005). Language, society and consciousness. Londres: Equinox.

Hyland, K. (2002). Genre: Language, context, and literacy. Annual Review of Applied Linguistics, 22, 113-135. 
Martin, J. (1991). Nominalization in science and humanities: Distilling knowledge and scaffolding text. En E. Ventola (Ed.), Functional and systemic linguistics (pp. 307338). Berlín: Mouton.

Martin, J. (1997). Analyzing genre: Functional parameters. En F. Christie \& J. Martin (Ed.), Genre and institutions: Social processes in the workplace and school (pp. 3-39). Londres: Continuum.

Martin, J. \& Rose, D. (2007). Working with discourse: Meaning beyond the clause. Londres: Continuum.

MEN (2004). Formar en ciencias: ¡El desafí!! Lo que necesitamos saber y saber hacer. Bogotá: Ministerio de Educación Nacional.

Mizuno, J. (2015). El lenguaje de la ciencia. En N. Barletta \& D. Chamorro (Eds.), El texto escolar y el aprendizaje: Enredos y desenredos (pp. 19-39). Barranquilla: Editorial Universidad del Norte.

Montenegro, A., Eastman, J., Mejía, G., Urrego, M., Rojas, I., Florián, I. \& Feo, J. (1994). Civilización 8. Bogotá: Norma.

Moss, G. (2010). Textbook language, ideology, and citizenship. Functions of Language, 17(1), 71-93.

Moss, G. (2013). Formas de construir conocimiento: Transitividad, ergatividad, metáfora gramatical y coherencia en textos escolares de Ciencias Naturales y Sociales. Lenguas en Contexto, 10, 5-16.

Moss, G., Natale, L. \& Oteíza, T. (2009). El lenguaje de los textos escolares, el aprendizaje, la ideología y la formación ciudadana. Revista D.E.L.T.A. 25, Número Especial, 641-677.

Moss, G., Barletta, N., Chamorro, D. \& Mizuno, J. (2013). La metáfora gramatical en los textos escolares de Ciencias Sociales en español. Onomazein, 28, 88-104 DOI 10.7764/onomazein.28.3

Moss, G., Mizuno, J., Ávila, D., Barletta, N., Carreño, S., Chamorro, D. \& Tapia C. (2003). Urdimbre del texto escolar: ¿Por qué resultan difíciles algunos textos? Barranquilla: Ediciones Uninorte.

Negrín, M. (2009). Los manuales escolares como objeto de investigación. Educación, Lenguaje y Sociedad, 6(6), 187-208.

Oteíza, T. (2006). El discurso pedagógico de la historia. Un análisis lingüistico sobre la construcción ideológica de la historia de Chile (1970-2001). Santiago de Chile: Frasis. 
Oteíza, T. (2011). Representación de las memorias del pasado: Intersubjetividad e el discurso pedagógico de la historia. En T. Oteíza \& D. Pinto (Eds.), En (re) construcción: Discurso, identidady nación en los manuales escolares de historia y de ciencias sociales (pp. 129-172). Santiago: Editorial Cuarto Propio.

Painter, C. (1996). The development of language as a resource for thinking: A linguistic view of learning. En R. Hasan \& G. Williams (Eds.), Literacy in Society (pp. 50-85). Londres: Addison Wesley Longman.

Ramos, C. \& Espinosa, O. (1997). Milenio 8: Historia y Geografía. Bogotá: Norma.

Rose, D. (1998). Science discourse and industrial hierarchy. En J. Martin, \& R. Veel (Eds.), Reading science: Critical and functional perspectives on discourses of science (pp. 236-265). Londres: Routledge.

Rose, D. (2006). Literacy and equality in the classroom. En A. Simpson (Ed.), Proceedings of the National Conference on Future Directions in Literacy. Sydney: University of Sydney [en línea]. Disponible en: http://sydney.edu.au/education_social_work/professional_learning/resource s/2006 papers.shtml

Rose, D. \& Acevedo, C. (2006). Literacy learning: The middle years. Australian Journal of Language and Literacy, 14(2), 32-45.

Schleppegrell, M., Achugar, M. \& Oteíza, T. (2004). The grammar of history: Enhancing content-based instruction through a functional focus on language. TESOL Quarterly, 38(1), 67-93.

Seco, O. \& Ramos, O. (1999). Diccionario del español actual. Madrid: Aguilar.

Short, D. (1994). Expanding middle school horizons: Integrating language, culture and social studies. TESOL Quarterly, 28, 581-608.

Unsworth, L. (1999). Developing critical understanding of the specialised language of school science and history texts: A functional grammatical perspective. Journal of Adolescent and Adult Literacy, 42, 508-521.

Vallejo, P. (2004). (Comp.). El discurso científico pedagógico: Aspectos de la textualización del saber enseñando. Bahía Blanca, Argentina: Editorial de la Universidad del Sur.

Vygotsky, L. (1986). Thought and language. Cambridge, Mass.: MIT Press.

Wignell, P. (1998). Technicality and abstraction in social science. En J. Martin \& R. Veel. (Eds.), Reading Science. Critical and functional perspectives on discourses of science (pp. 297-326). Londres: Routledge. 
Williams, G. (1999). The pedagogic device and the production of pedagogic discourse: A case example in early literacy education. En F. Christie (Ed.), Pedagogy and the shaping of consciousness: Linguistic and social processes (pp. 82-122). Londres: Cassell.

\section{NOTAS}

${ }^{1} \mathrm{El}$ artículo es producto de la investigación: "Comparación de los rasgos discursivos de dos textos escolares de Ciencias Sociales (1999, 2010)”, auspiciada por la Dirección de Investigación, Desarrollo e Innovación de la Universidad del Norte, Barranquilla, Colombia.

${ }^{2}$ Los manuales escolares de los cuales se toman los ejemplos se identifican con la inicial del nombre del texto, seguido del número de la página: CS: Ciencias Sociales 8; M: Milenio 8; H: Hipertexto 8. ; C: Civilización 8. 